\title{
Effects of rTMS and tDCS on neuropathic pain after brachial plexus injury: A randomized placebo- controlled pilot study
}

Erickson Duarte Bonifácio de Assis

Federal University of Paraíba

Wanessa Kallyne Nascimento Martins

Federal University of Paraíba

Carolina Dias de Carvalho

Federal University of Paraíba

Clarice Martins Ferreira

Federal University of Paraíba

Ruth Gomes

Federal University of Paraíba

Evelyn Thais Almeida Rodrigues

Federal University of Paraíba

Ussânio Mororó Meira

Federal University of Paraíba

Edgard Morya

Santos Dumont Institute

Suellen Marinho Andrade ( $\sim$ suellenandrade@gmail.com )

Federal University of Paraíba

\section{Research Article}

Keywords: Repetitive Transcranial Magnetic Stimulation, Transcranial Direct-Current Stimulation, Pain, Peripheral Nervous System Diseases

Posted Date: May 11th, 2021

DOI: https://doi.org/10.21203/rs.3.rs-456578/v1

License: (c) (i) This work is licensed under a Creative Commons Attribution 4.0 International License.

Read Full License 


\section{ORIGINAL RESEARCH}

\section{Effects of rTMS and tDCS on neuropathic pain after brachial plexus injury:}

\section{A randomized placebo-controlled pilot study}

Erickson Duarte Bonifácio de Assis ${ }^{a}$, Wanessa Kallyne Nascimento Martins ${ }^{\mathrm{a}}$, Carolina Dias de Carvalho ${ }^{\mathrm{a}}$, Clarice Martins Ferreira ${ }^{\mathrm{a}}$, Ruth Gomes ${ }^{\mathrm{a}}$, Evelyn Thais de Almeida Rodrigues ${ }^{\mathrm{a}}$, Ussânio Mororó Meira ${ }^{\mathrm{a}}$, Edgard Morya ${ }^{\mathrm{b}}$, Suellen Marinho Andrade $\mathrm{a}^{\mathrm{a}^{*}}$

${ }^{a}$ Aging and Neuroscience Laboratory, Federal University of Paraíba, João Pessoa, Brazil

${ }^{\mathrm{b}}$ Edmond and Lily Safra International Institute of Neuroscience, Santos Dumont Institute, Macaíba, Rio Grande do Norte, Brazil.

* Corresponding author: Suellen Marinho Andrade. Aging and Neuroscience Laboratory, Federal University of Paraíba, 58052-900 João Pessoa, Paraíba, Brazil. Tel.: +558332098756; Fax.: +558332098756. E-mail address: suellenandrade@gmail.com. Institutional URL. http://www.cchla.ufpb.br/ppgnec/en/

Clinical Trial Registration: http://www.ensaiosclinicos.gov.br/, RBR-5xnjbc - Sep 3, 2018 


\title{
Effects of rTMS and tDCS on neuropathic pain after brachial plexus injury:
}

\section{A randomized placebo-controlled pilot study}

\begin{abstract}
Neuropathic pain (NP) after brachial plexus injury (NPBPI) is a highly disabling clinical condition and is increasingly prevalent due to increased motorcycle accidents. Currently, no randomized controlled trials have evaluated the effectiveness of non-invasive brain stimulation techniques such as Repetitive Transcranial Magnetic Stimulation (rTMS) and Transcranial Direct-Current Stimulation (tDCS) in patients suffering from NPBPI. In this study, we directly compare the efficacy of 10-Hz rTMS and anodal $2 \mathrm{~mA}$ tDCS techniques applied over the motor cortex (5 daily consecutive sessions) in 21 patients with NPBPI, allocated into 2 parallel groups (active or sham). The order of the sessions was randomised for each of these treatment groups according to a crossover design and separated by a 30-day interval. Scores for "continuous" and "paroxysmal" pain (primary outcome) were tabulated after the last stimulation day and 30 days after. Secondary outcomes included the improvement in multidimensional aspects of pain, anxiety state and quality of life. Active rTMS and tDCS were both superior to sham in reducing continuous $(\mathrm{p}<0,001)$ and paroxysmal pain $(\mathrm{p}=0.04)$ as well as in multidimensional aspects of pain and anxiety state. Repetitive TMS was superior to tDCS in reducing continuous $(\mathrm{p}=0.01)$ and paroxysmal pain $(\mathrm{p}=0.03)$, and in improving multidimensional aspects of pain $(\mathrm{p}=0.01)$. Our results suggest rTMS and tDCS are able to treat NPBPI with little distinction in pain and anxiety state, which may promote the use of tDCS in brachial plexus injury pain management, as it constitutes an easier and more available technique.
\end{abstract}

Keywords: Repetitive Transcranial Magnetic Stimulation; Transcranial Direct-Current Stimulation; Pain; Peripheral Nervous System Diseases. 


\section{Introduction}

Traumatic brachial plexus injuries are diagnosed in more than $1 \%$ of patients treated in emergency units ${ }^{1,2}$. Neuropathic pain after brachial plexus injury affects 30 to $90 \%$ of patients $^{3-7}$, and occurs due to deafferentation, i.e. a loss of sensory afferent input, most commonly in preganglionic lesions when there is brachial plexus avulsion or in complete lesions $^{8,9}$. The pain is usually severe, mainly located in the forearm and hand, in the form of a continuous burning sensation associated with acute pain paroxysms ${ }^{10,11}$. Since chronic pain results from inadequate plastic changes in the central and peripheral nervous system ${ }^{12}$, noninvasive brain stimulation techniques such as repetitive transcranial magnetic stimulation (rTMS) and transcranial direct-current stimulation (tDCS) have been has been reported as a therapeutic option ${ }^{12-14}$.

Recent neurophysiological support and previous neuroimaging studies for this possibility comes from reports that rTMS and tDCS over functionally connected regions of the distributed motor network leads to greater enhancements in pain relief, especially given the evidence to modulate the activity of an extensive neuronal network ${ }^{13}$, which includes thalamic nuclei, the limbic system, brain stem nuclei, and spinal medulla ${ }^{15-18}$. However, currently, a direct comparison approach about rTMS and tDCS techniques has not been explored in relation to neuropathic pain located in the upper limb $b^{12,14,18}$.

Methodological challenges in pairwise treatment comparisons are likely due to potential effect modifiers such as inter-individual variability in each participant enrolled in a parallel arm designs and complex differences of tDCS and rTMS parameters.

In the current crossover preliminary study, we focused on comparing changes in pain intesity and multidimensional aspects from patients with NPBPI. In light of previous experiments demonstrating changes in cortical activity following rTMS under different painful conditions ${ }^{12,19,20}$, we hypothesised that rTMS over the primary motor cortex would modulate motor network excitability more effectively than tDCS in NPBPI individuals, and produce greater clinical changes, due to the additional motor pathways emerging from rTMS. Lastly, as a secondary analysis, we explored the effects of stimulation over the anxiety and quality of life in this population, which constitute two important nodes within the management of chronic pain.

\section{Methods}

\section{Participants}


Participants were recruited from a referral centre in pain management, between September and December 2018. Patients aged between 18 and 60 years who scored 4 to 10 points on the Visual Analogue Scale (VAS), with NPBPI refractory to clinical treatment, persistent for at least 6 months, and who received adequate pharmacological treatment for pain with the combination of antidepressants, gabapentinoid antiepileptics and analgesic opioids $^{21}$, for at least 1 month before the study were considered eligible ${ }^{13}$. We applied the DN4 Questionnaire to confirm the presence of neuropathic pain $(\mathrm{NP})^{22,23}$. No changes in medication regimens were allowed during the study. Exclusion criteria were the presence of other neurological or psychiatric diseases, including major ongoing depression, history of substance abuse, in addition to formal contraindications for rTMS and/or tDCS ${ }^{12,14}$. We applied the Beck Depression Inventory (BDI ${ }^{24}$ to identify and grade depressive symptoms. The selected participants underwent rTMS and tDCS sessions conducted in an institutional neuromodulation laboratory.

\section{Ethical aspects}

This study was carried out respecting the ethical principles expressed in the Declaration of Helsinki ${ }^{25}$, and all participants voluntarily signed an informed consent form. The protocol was previously approved by the Research and Ethical Committee of the Health Sciences Center at Federal University of Paraíba (statement 2.563.783), and was registered at ClinicalTrials (ensaiosclinicos.gov.br) with the ID number RBR-5xnjbc (03/09/2018).

\section{Study design}

We conducted a pilot, placebo-controlled, double-blind, randomized, crossover clinical trial in accordance with the CONSORT guidelines ${ }^{5,26}$. Participants were randomly allocated to one of 2 parallel groups: active or sham stimulation, a ratio of 1:1. We used a random number generator in an online randomization program (www.random.org). The allocation was hidden using sequential numbered, opaque and sealed envelopes.

The procedures related to allocation, randomization, evaluation, intervention, and data analysis were carried out by independent researchers who were unaware of each other's work. Blinding was also extended to patients, who were not aware of the allocation group and hypotheses of the study.

The session order (rTMS and tDCS) was random for each treatment group (active or sham) according to a crossover design. Group 1 received active rTMS followed by active 
tDCS or active tDCS followed by active rTMS, while group 2 received sham rTMS followed by sham tDCS or sham tDCS followed by sham rTMS (Fig. 1). This design was based on a previous clinical study ${ }^{13}$, and avoided the need for patients to receive placebo and active stimulation in the same crossed arm. The treatment protocol included 2 stimulation blocks separated by a 30-day interval, a period of washout considered sufficient to reduce possible carry over effects and compatible with that reported in a previous study ${ }^{13,27,28}$. Each block consisted of 5 sessions for 5 consecutive days, during which each patient received rTMS or tDCS for 30 minutes. At the end, each patient received a total of 10 stimulation sessions (2 series of active rTMS/tDCS or 2 series of sham rTMS/tDCS; Fig. 1).

\section{Insert Figure 1}

\section{Assessments and Outcomes}

In the baseline visit we perform the structured questionnaire included demographic data, affected side, injury characteristics, DN4 and BDI scores, and current clinical treatment. Primary and secondary outcomes were developed in accordance with IMMPACT recommendations for clinical trials related to chronic pain treatment ${ }^{29}$.

The primary outcome was the improvement in pain intensity measured by the Visual Analogue Scale (VAS) ${ }^{30}$. Scores for "continuous pain" and "paroxysmal pain"10,31 for the last 24 hours were obtained. Assessments were performed. in each stimulation block, immediately before the first stimulation session $\left(\mathrm{T}_{0}\right)$, after the $5^{\text {th }}$ consecutive stimulation session $\left(\mathrm{T}_{1}\right)$ and after a 30-day interval $\left(\mathrm{T}_{2}\right)$.

Secondary outcomes were concurrently assessed with the primary outcome as follows: (1) multidimensional aspect of pain based on the McGill Pain Questionnaire (MPQ) ${ }^{32}$; (2) the anxiety state measured by the State-Trait Anxiety Inventory (state subscale) (STAI-S) ${ }^{33}$; and (3) changes in quality of life assessed by the SF-36 Quality of Life Questionnaire ${ }^{34}$. The safety of rTMS and tDCS was assessed by monitoring the occurrence of adverse effects during treatment, with the application of a checklist at the end of each session.

\section{Interventions}

\section{Repetitive transcranial magnetic stimulation}

The TMS device was a Neuro-MS / D magnetic stimulator (Neurosoft Ltd., Ivanovo, Russia), using an angled and cooled figure-of-8 coil (F8) over M1 contralateral to the painful 
side. The rTMS parameters were similar to those reported in previous studies ${ }^{18,12}$ : $90 \%$ RMT, $10 \mathrm{~Hz}, 2500$ pulses per session (25 trains of 10 seconds each, with an interval of 17 seconds) on 5 consecutive days.

The ideal coil position was marked on an elastic cap worn in each session, to signal the target area in M1. This marking was based on: (1) the International 10/20 System; (2) surface anatomy references; and (3) resting motor threshold (RMT) record. The coil was held in position by an articulated support, tangentially oriented to the interhemispheric fissure, and all subjects clearly heard the coil noise.

The M1 hot spot was defined according to the motor function grade in the affected limb. We applied a single TMS pulse in patients with useful hand motor function to elicit a minimal visible contraction in a muscle of the fingers (i.e. abductor pollicis or first dorsal interosseous) in at least 5 of 10 pulses $^{35,36}$. We used hemiface muscles ipsilateral to the painful limb as reference in patients without useful motor function due to the somatotopic cortical proximity in relation to the motor area of the hand ${ }^{19,37,38}$.

The procedures for locating M1 and determining RMT were the same for the rTMS applied in the placebo group. The same F8 coil, but elevated and tilted out of the head, was used to reproduce some of the subjective sensation of rTMS to simulate rTMS sessions, and still avoid current induction in the brain ${ }^{17,30,35-40}$. Since none of the patients had previously experienced rTMS, they had no idea what an active stimulus would feel like $17,30,35-40$.

\section{Transcranial direct-current stimulation}

A battery-powered tDCS stimulator (TCT Research Ltd., Kowloon, Hong Kong) was used. The stimulation protocol was guided by previous studies ${ }^{14,41}$, with the active current applied in M1 contralateral to the painful side. The anode was positioned over C3 or C4 according to (1) the International 10/20 System and (2) surface anatomy references, and the cathode positioned over supraorbital region contralateral to the anode ${ }^{42}$. The electrodes were wrapped by $5 \times 7 \mathrm{~cm}$ sponges, moistened with saline $(\mathrm{NaCl} 0.9 \%)$, with an applied current of $2 \mathrm{~mA}$, and the current density equivalent to $0.057 \mathrm{~mA} / \mathrm{cm}^{2}$.

The protocol for tDCS placebo was identical, but the device stopped emitting a current 30 seconds after starting the stimulation. Thus, the effects of active stimulation (slight tingling and itching sensation) were simulated, constituting a reliable blinding method with the effects disappearing soon after the stimulation started ${ }^{41}$.

\section{Statistical analysis}


The analyses were based on the intent-to-treat principle. Clinical and demographic variables were compared at baseline through one-way analysis of variance (ANOVA) for continuous data, or the Chi-squared test for categorical data.

All efficacy measures, corresponding to the primary outcome (mean score of continuous and paroxysmal pain intensity) and all secondary efficacy variables (MPQ scores, STAI-S and SF-36), were analysed by a split plot ANOVA with a mixed effects model. The model included the following explanatory variables: group (rTMS, tDCS or sham stimulation), time ( $\mathrm{T}_{0}, \mathrm{~T}_{1}$ and $\mathrm{T}_{2}$ ), order of the sessions (rTMS followed by tDCS or tDCS followed by rTMS), and the interaction effect between group and time. The baselineobservation-carried-forward (BOCF) approach was used to handle missing data.

Comparisons between groups were considered post hoc and corrected by the Bonferroni procedure. Fisher's exact test was used to compare proportions. P-values $<0.05$ were considered significant in all cases. The effect size was obtained through partial etasquared and Cohen's d according to each type of comparison. All analyses were performed by an independent researcher with the IBM SPSS Statistics 22.0 computer program (IBM Corp., Armonk, NY).

\section{Results}

\section{Patients}

We screened 27 patients with NPBPI and refractory to treatment, all of them male; 6 patients were excluded for not meeting the eligibility criteria or refusal (Fig. 2). The remaining 21 patients were randomly allocated to groups of active stimulation $(n=12)$ or passive stimulation $(n=9)$, but one patient withdrew from the trial before the first session was conducted. Data were obtained for 20 patients who underwent at least one active or sham stimulation session (Fig. 2).

\section{Insert Figure 2}

Table 1 presents the baseline demographic and clinical characteristics of the participants such as affected side, injury characteristics, DN4 questionnaire, BDI scores, current clinical treatment, pain intensity scores (VAS), MPQ, STAI-S and SF-36. These characteristics were not significantly different between the groups at baseline $\left(T_{0}\right)(p>0.05)$ (Table 1). 
No differences were found between the subgroups of sham stimulation (rTMS and tDCS) $(p>0.05)$, and therefore we combined them to form a single sham-stimulation group. In this sense, 3 groups were considered in the analysis of the results: active rTMS, active tDCS and sham stimulation.

\section{Primary outcome}

\section{Continuous pain}

The comparison of changes in continuous pain intensity between rTMS, tDCS and sham stimulation showed a significant group $\left(F=11.76 ; p<0.001 ; n_{p}{ }^{2}=0.56\right)$ and time effect $\left(\mathrm{F}=10.08 ; \mathrm{p}=0.02 ; \mathrm{n}_{\mathrm{p}}{ }^{2}=0.42\right)$, with significant interaction between group and time $(\mathrm{F}$ $=3.18 ; \mathrm{p}=0.01)$, indicating that both $\mathrm{rTMS}$ and tDCS are superior to sham stimulation $(\mathrm{p}<$ 0.04). However, rTMS was able to induce significantly stronger analgesic effects than tDCS and sham stimulation ( $\mathrm{rTMS}$ versus sham stimulation: $\mathrm{t}=9.11 ; \mathrm{p}=0.02 ; \mathrm{d}=0.81 ; \mathrm{tDCS}$ versus sham stimulation: $\mathrm{t}=10.43 ; \mathrm{p}=0.03 ; \mathrm{d}=0.76$ ). More specifically, there was a small advantage in relation to the effect size when comparing rTMS and tDCS (rTMS versus tDCS: $\mathrm{t}=8.09 ; \mathrm{p}=0.01 ; \mathrm{d}=0.21)$.

Paired comparisons after the 30-day interval $\left(\mathrm{T}_{2}\right)$ showed that $\mathrm{rTMS}(\mathrm{t}=7.02 ; \mathrm{p}=$ $0.02 ; d=0.72)$ and tDCS $(t=5.64 ; p=0.04 ; d=0.68)$ were more effective than the sham stimulation, but no differences between both active techniques were found $(t=0.29 ; p=1.47$; $\mathrm{d}=0.10)($ Fig. 3).

\section{Insert Figure 3}

\section{Paroxysmal pain}

According to Figure 4, the comparison of changes in paroxysmal pain intensity between rTMS, tDCS and sham stimulation showed a group effect $\left(F=18.46 ; p=0.04 ; n_{p}^{2}=\right.$ $0.61)$, but not a time effect $\left(F=0.97 ; p=2.49 ; n_{p}^{2}=0.16\right)$. The efficacy of active stimulations (rTMS and tDCS) was confirmed after the last session $\left(\mathrm{T}_{1}\right)$ in comparison to the sham stimulation group ( $\mathrm{p}<0.05$ ). Paired comparisons revealed a higher effect of rTMS on tDCS, with a moderate effect size $(t=4.76 ; p=0.03 ; d=0.31)$. No difference was observed between the three types of stimulation after the 30-day interval $\left(\mathrm{T}_{2}\right)$ (rTMS, tDCS and sham stimulation $)\left(F=2.36 ; p=1.19 ; n_{p}^{2}=0.07\right)($ Fig. 4). 


\section{Insert Figure 4}

\section{Secondary outcomes}

\section{Multidimensional aspect of pain}

A significant group effect $\left(F=15.28 ; p=0.01 ; n_{p}^{2}=0.73\right)$ was observed regarding the scores achieved in evaluating the multidimensional aspect of pain (MPQ). A significant effect of active stimulations (rTMS and tDCS) after the last session $\left(\mathrm{T}_{1}\right)$ was observed, which was superior in the rTMS group when compared to tDCS $(\mathrm{t}=2.14 ; \mathrm{p}=0.01 ; \mathrm{d}=0.48)$ and sham stimulation $(t=8.23 ; p=0.02 ; d=0.82)$. Transcranial DCS was also superior, although to a lesser effect, to sham stimulation $(\mathrm{t}=8.13 ; \mathrm{p}=0.03 ; \mathrm{d}=0.71)$. However, after the 30-day interval $\left(\mathrm{T}_{2}\right)$, no difference was observed between the three types of stimulations $(\mathrm{p}>0.05)$ (Fig. 5).

\section{Insert Figure 5}

\section{Anxiety state}

A significant group effect $\left(\mathrm{F}=9.73 ; \mathrm{p}=0.04 ; \mathrm{n}_{\mathrm{p}}{ }^{2}=0.43\right)$ regarding the scores achieved in the anxiety state assessment was observed. Repetitive TMS $(t=8.93 ; p=0.02 ; d$ $=0.81)$ and $\operatorname{tDCS}(\mathrm{t}=10.28 ; \mathrm{p}=0.01 ; \mathrm{d}=0.85)$ were more effective than the sham stimulation after the last session $\left(\mathrm{T}_{1}\right)$ in the STAI-S (Fig. 6) measurements. Paired comparisons showed no differences between active techniques $(t=0.98 ; p=2.16 ; d=0.08)$. The achieved improvement levels were maintained after the 30-day interval $\left(\mathrm{T}_{2}\right)$ for both active stimulations $(\mathrm{p}<0.05)$.

\section{Insert Figure 6}

\section{Quality of life}

No difference was observed between the three types of stimulations regarding scores achieved in quality of life (SF-36) throughout the course of treatment ( $p>0.05)$.

\section{Safety}

The proportion of patients displaying side effects was low and similar between the groups $(\mathrm{p}>0.05)$ (Table 2). No patients withdrew from the treatment because of such effects. 
Increase in pain scores was observed in some patients in all of the active e sham groups. Autonomic dysfunctions such as limb edema and worsening of Horner's syndrome were reported in the active tDCS group.

\section{Insert Table 2}

\section{Discussion}

In this preliminary study, we performed a double-blind, crossover and controlled investigation comparind rTMS versus tDCS effects in pain management. To our knowledge, this is the first known study to compare these two methods of non-invasive brain stimulation in neuropathic pain located in the upper limb ${ }^{12,14,18}$. Overall, we observed that rTMS and tDCS applied in M1 are both effective in reducing continuous and paroxysmal pain after brachial plexus injury. Such analgesic effect also promoted improvement in multidimensional aspects of pain, anxiety-state, but not in quality of life. However, rTMS resulted in greater improvements after the endpoint.

Repetitive TMS and tDCS aim to induce depolarization mechanisms in an attempt to reduce chronic pain, directly altering brain activity in an extensive neuronal network involved in pain processing ${ }^{43}$. Our results suggest similar modulation mechanisms are involved in reducing pain after brachial plexus injury, although the areas and pathways involved in each technique may be distinct, including the effect on the subtype of pain - continuous burning pain and paroxysmal shooting pain. The distinction between these NP patterns in this population is common in clinical practice and possibly involves different pathophysiological mechanisms $6,10,31,44$.

The finding that rTMS has a particularly superior impact on pain deserves further investigation into the mechanisms of action. Intriguingly, previous report indicates that epidural motor cortex stimulation (MCS) for BPA pain was ineffective for paroxysmal pain but moderately effective for continuous pain $^{44}$. In BPA, paroxysmal pain is thought to originate from hyperactive neurons in the dorsal horn, whereas continuous pain is thought to originate from supraspinal structures, particularly the thalamus ${ }^{6,44}$. Nevertheless, neuronal hyperactivity has been also detected in thalamic nuclei, suggesting that supraspinal mechanisms contribute to paroxysmal shooting pain generation ${ }^{45}$. Furthermore, antinociceptive effects of rTMS, similar to MCS, can be mediated by the corticotalamic tract regardless of the functional integrity of the lemniscal system descending from the brainstem 
to the spinal cord (often compromised in BPA patients) ${ }^{6,9,46}$, explained not only the improvement in continuous pain but also in paroxysmal pain verified in rTMS active group.

Likewise, we obtained a significant result of tDCS in improving NP which affects the upper limb after brachial plexus injury. Regarding action mechanisms, up-regulation of motor cortex excitability by tDCS can induces remote indirect effects, not only in the thalamus, but, in particular, in the prefrontal and parietal areas ${ }^{13,31,47,48}$. However, few studies on $\mathrm{NP}^{14,18}$ proved to only be effective against individuals with lower-limb NP, such as that due to diabetic polyneuropathy or spinal cord injury when an anodal current of $2 \mathrm{~mA}$ was applied over the left M1 or contralaterally to the painful side $\mathrm{s}^{31,49,50}$. It is probable that BPA affects mainly the central nervous system structures than peripheral one ${ }^{9}$. Animal models of avulsion led to a more pronounced injury to the medial aspect of the Lissauer tract and the lateral dorsal column, with subsequent gliosis of the substantia gelatinosa that are closer to spinal cord lesions than to post-ganglionic injuries ${ }^{9,51,52}$. In addition, a positron emission tomography study has shown significantly increased metabolism after active tDCS in the medulla in patients with neuropathic pain after spinal cord injury ${ }^{50}$. These mixed-mechanisms data may corroborate the pain relief achieved in our study.

Interestingly, rTMS was superior in improving multidimensional aspects of pain (MPQ). Based on previous evidence with MCS, multiple-session high-frequency rTMS over M1 is similarly capable to trigger a cascade of events of long time course involving perigenual cingulate and orbitofrontal areas, whose are considered critical for modulate the emotional appraisal of pain ${ }^{12,16}$. On the other hand, transcranial DCS also shows benefits in MPQ scores, although with smaller effect size. Possibly, anodal stimulation over M1 might modulate emotional and cognitive components of pain and normalize excessive attention to pain and pain-related information ${ }^{50}$.

The active treatment groups of both techniques showed better results compared to the placebo group in relation to anxiety state (STAI-S) and maintaining this improvement in the medium-term (30-day follow-up). Although, anxiety associated with other neurological disorders ${ }^{53}$ such as BPI associated with chronic pain ${ }^{11}$ can negatively impact the quality of life, we do not obtained improvement in SF-36 scores. Indeed, the expected maintenance of physical disability throughout the course of treatment with NIBS techniques ${ }^{43}$ probably contributed to not improving the quality of life. However, these results should be interpreted with caution and corroborated in future studies, including a longer time of stimulation and follow-up. 
Although both techniques are non-invasive, tDCS has a lower cost, easier technical execution and more portability when compared to $\mathrm{rTMS}^{20}$, which has probably led to a growing increase in clinical research on tDCS over the last few years ${ }^{14}$, despite well-defined evidence for rTMS in NP ${ }^{12,18}$. In this sense, even with the superiority of rTMS over tDCS, we obtained a favourable short-term result for both techniques in pain aspects and anxiety state, as well as differences in the 30-day follow-up for continuous pain intensity, when compared to the sham stimulation; Perhaps, a more accurate and thorough knowledge of the correlation between the symptomatology and the pathophysiology of pain subtypes in brachial plexus injury might most certainly lead to further clinical progress and help in choosing the technique, since rTMS is not always available, which may provide greater use of tDCS in future clinical practice.

Our study has limitations which should be acknowledged. We did not employ neuroimaging and computational modelling techniques to control cortical changes or possible interference related to the disease neurophysiology. In order to minimize this bias, we controlled the eligibility criteria and randomized the groups and the order of sessions. Another limitation refers to the number of participants and the number of sessions performed. group.

\section{Conclusions}

Finally, the applicability of NIBS in this type of pain syndrome should be reproduced and better evaluated in future clinical trials with a larger number of participants and sessions in order to verify a long-lasting pain relief result in association with improvement in quality of life. Notwithstanding the above, our results highlight the potential use of tDCS for chronic pain management in traumatic brachial plexus injuries with little distinction from rTMS, which may promote the use of an easier and more available technique as part of an interdisciplinary approach in rehabilitation services for patients following upper limb deafferentation.

\section{Disclosure}

The authors have nothing to disclose. All the authors had full access to all the data and took final responsibility for the decision to submit for publication.

\section{Data availability}

The datasets generated during and analysed during the current study are not publicly available but are available from the corresponding author on reasonable request. 


\section{References}

1. Faglioni, W., Martins, R. S., Heise, C. O., \& Foroni, L. The epidemiology of adult traumatic brachial plexus lesions in a large metropolis. Acta Neurochirurgica 156, 10251028. https://doi.org/10.1007/s00701-013-1948-x (2014).

2. Midha, R. Epidemiology of brachial plexus injuries in a multitrauma population. Neurosurgery 40, 1182-1189. https://doi.org/10.1097/00006123-199706000-00014 (1997).

3. Ciaramitaro, P., et al. Traumatic peripheral nerve injuries: Epidemiological findings, neuropathic pain and quality of life in 158 patients. Journal of the Peripheral Nervous System, 15, 120-127. https://doi.org/10.1111/j.1529-8027.2010.00260.x (2010).

4. Flores, L. P. Estudo epidemiológico das lesões traumáticas de plexo braquial em adultos. Arquivos de Neuro-Psiquiatria 64, 88-94. https://doi.org/10.1108/00035590610650785 (2006).

5. Moher, D., et al. CONSORT 2010 explanation and elaboration: updated guidelines for reporting parallel group randomised trials. BMJ (Clinical Research Ed.) 340, c869. https://doi.org/10.1136/bmj.c869 (2010).

6. Sindou, M. P., Blondet, E., Emery, E., \& Mertens, P. Microsurgical lesioning in the dorsal root entry zone for pain due to brachial plexus avulsion: a prospective series of 55 patients. Journal of Neurosurgery 102, 1018-1028. https://doi.org/10.3171/jns.2005.102.6.1018 (2005).

7. Treede, R.-D., et al. Neuropathic pain: redefinition and a grading system for clinical and research purposes. Neurology 70, 1630-1635.

https://doi.org/10.1212/01.wnl.0000282763.29778.59 (2008).

8. Ferreira, K. A., Teixeira, M. J., Mendonza, T. R., \& Cleeland, C. S. Validation of brief pain inventory to Brazilian patients with pain. Supportive Care in Cancer 19, 505-511. https://doi.org/10.1007/s00520-010-0844-7 (2011).

9. Teixeira, M. J., et al. Neuropathic pain after brachial plexus avulsion - central and peripheral mechanisms. BMC Neurology 15, 73. https://doi.org/10.1186/s12883-015-0329-x (2015).

10. Parry, C. B. W. Pain in avulsion lesions of the brachial plexus. Pain 9, 41-53. https://doi.org/10.1016/0304-3959(80)90027-5 (1980).

11. Santana, M. V. B., et al. High prevalence of neuropathic pain in the hand of patients with traumatic brachial plexus injury: a cross-sectional study. Arquivos de Neuro-Psiquiatria 74, 895-901. https://doi.org/10.1590/0004-282X20160149 (2016).

12. Lefaucheur, J. P., et al. Evidence-based guidelines on the therapeutic use of repetitive transcranial magnetic stimulation (rTMS). Clinical Neurophysiology 125, 2150-2206. https://doi.org/10.1016/j.clinph.2014.05.021 (2014).

13. Attal, N., et al. Repetitive transcranial magnetic stimulation and transcranial direct-current stimulation in neuropathic pain due to radiculopathy: a randomized sham-controlled comparative study. Pain 157, 1224-1231. https://doi.org/10.1097/j.pain.0000000000000510 (2016). 
14. Lefaucheur, J. P., Antal, A., \& Ayache, S. Evidence-based guidelines on the therapeutic use of transcranial direct current stimulation (tDCS). Clinical Neurophysiology 128, 56-92. https://dx.doi.org/10.1016/j.clinph.2016.10.087 (2017).

15. Cruccu, G., et al. EAN guidelines on central neurostimulation therapy in chronic pain conditions. European Journal of Neurology 23, 1489-1499.

https://doi.org/10.1111/ene.13103 (2016).

16. Garcia-Larrea, L., \& Peyron, R. Motor cortex stimulation for neuropathic pain: From phenomenology to mechanisms. NeuroImage 37, S71-S79.

https://doi.org/10.1016/j.neuroimage.2007.05.062 (2007).

17. Holsheimer, J., Nguyen, J. P., Lefaucheur, J. P., \& Manola, L. Cathodal, anodal or bifocal stimulation of the motor cortex in the management of chronic pain? Acta Neurochirurgica Supplementum 97, 57-66. https://doi.org/10.1007/978-3-211-33081-4_7 (2007).

18. Baptista, A. F., et al. Latin American and Caribbean consensus on noninvasive central nervous system neuromodulation for chronic pain management (LAC 2 -NIN-CP). Pain Reports 4, 1-20. https://doi.org/10.1097/PR9.0000000000000692 (2019).

19. Lefaucheur, J. P., et al. Neurogenic pain relief by repetitive transcranial magnetic cortical stimulation depends on the origin and the site of pain. Journal of Neurology, Neurosurgery and Psychiatry 75, 612-616. https://doi.org/10.1136/jnnp.2003.022236 (2004).

20. Priori, A., Hallett, M., \& Rothwell, J. C. Repetitive transcranial magnetic stimulation or transcranial direct current stimulation? Brain Stimulation 2, 241-245. https://doi.org/10.1016/j.brs.2009.02.004 (2009).

21. Dworkin, R. H., et al. Recommendations for the pharmacological management of neuropathic pain: An overview and literature update. Mayo Clinic Proceedings $\mathbf{8 5}$. https://doi.org/10.4065/mcp.2009.0649 (2010).

22. Baron, R., Binder, A., \& Wasner, G. Neuropathic pain: Diagnosis, pathophysiological mechanisms, and treatment. The Lancet Neurology 9, 807-819. https://doi.org/10.1016/S1474-4422(10)70143-5 (2010).

23. Bouhassira, D., et al. Comparison of pain syndromes associated with nervous or somatic lesions and development of a new neuropathic pain diagnostic questionnaire (DN4). Pain 114, 29-36. https://doi.org/10.1016/j.pain.2004.12.010 (2005).

24. Beck, A., Steer, R., \& Brown, G. (1996). Beck depression inventory-II. Psychological Assessment 78, 490-498. https://doi.org/10.1037/t00742-000

25. World Medical Association. World Medical Association Declaration of Helsinki: Ethical principles for medical research involving human subjects. JAMA - Journal of the American Medical Association 310, 2191-2194. https://doi.org/10.1001/jama.2013.281053 (2013).

26. Eldridge, S. M., et al. CONSORT 2010 statement: extension to randomised pilot and feasibility trials. Pilot and Feasibility Studies 2, 64. https://doi.org/10.1186/s40814-016-01058 (2016).

27. Cervigni, M., et al. Repetitive transcranial magnetic stimulation for chronic neuropathic pain in patients with bladder pain syndrome/interstitial cystitis. Neurourology and Urodynamics 37, 2678-2687. https://doi.org/10.1002/nau.23718 (2018).

28. Palm, U., et al. Effects of transcranial random noise stimulation (tRNS) on affect, pain 
and attention in multiple sclerosis. Restorative Neurology and Neuroscience 34, 189-199. https://doi.org/10.3233/RNN-150557 (2016).

29. Dworkin, R. H., et al. Interpreting the Clinical Importance of Treatment Outcomes in Chronic Pain Clinical Trials: IMMPACT Recommendations. Journal of Pain 9, 105-121. https://doi.org/10.1016/j.jpain.2007.09.005 (2008).

30. Haefeli, M., \& Elfering, A. Pain assessment. European Spine Journal 15, S17-S24. https://doi.org/10.1007/s00586-005-1044-x (2006).

31. Soler, M. D., et al. Effectiveness of transcranial direct current stimulation and visual illusion on neuropathic pain in spinal cord injury. Brain 133, 2565-2577. https://doi.org/10.1093/brain/awq184 (2010).

32. Melzack, R. The McGill Pain Questionnaire: Major properties and scoring methods. Pain 1, 277-299. https://doi.org/10.1016/0304-3959(75)90044-5 (1975).

33. Spielberger, C. D. STAI manual for the state-trait anxiety inventory (Self-evaluation questionnare) in Consulting Psychogyists Press (Mind Garden, 1970).

34. Ware, J. E., \& Sherbourne, C. D. The MOS 36-item short-form health survey (Sf-36): I. conceptual framework and item selection. Medical Care 30, 473-483. https://doi.org/10.1097/00005650-199206000-00002 (1992).

35. Hanajima, R., et al. Comparison of different methods for estimating motor threshold with transcranial magnetic stimulation. Clinical Neurophysiology: Official Journal of the International Federation of Clinical Neurophysiology 118, 2120-2122. https://doi.org/10.1016/j.clinph.2007.05.067 (2007).

36. Hosomi, K., et al. Daily repetitive transcranial magnetic stimulation of primary motor cortex for neuropathic pain: A randomized, multicenter, double-blind, crossover, shamcontrolled trial. Pain 154, 1065-1072. https://doi.org/10.1016/j.pain.2013.03.016 (2013).

37. Ahmed, M. A., Mohamed, S. A., \& Sayed, D. Long-term antalgic effects of repetitive transcranial magnetic stimulation of motor cortex and serum beta-endorphin in patients with phantom pain. Neurological Research 33, 953-958.

https://doi.org/10.1179/1743132811Y.0000000045 (2011).

38. Kang, B. S., Shin, H. I., \& Bang, M. S. Effect of Repetitive Transcranial Magnetic Stimulation Over the Hand Motor Cortical Area on Central Pain After Spinal Cord Injury. Archives of Physical Medicine and Rehabilitation 90, 1766-1771.

https://doi.org/10.1016/j.apmr.2009.04.008 (2009).

39. Khedr, E. M., et al. Repetitive transcranial magnetic stimulation in neuropathic pain secondary to malignancy: A randomized clinical trial. European Journal of Pain 19, 519-527. https://doi.org/10.1002/ejp.576 (2015).

40. Khedr, E. M., et al. Longlasting antalgic effects of daily sessions of repetitive transcranial magnetic stimulation in central and peripheral neuropathic pain. Journal of Neurology, Neurosurgery and Psychiatry 76, 833-838. https://doi.org/10.1136/jnnp.2004.055806 (2005).

41. Nitsche, M. A., et al. Transcranial direct current stimulation: State of the art 2008. Brain Stimulation 1, 206-223. https://doi.org/10.1016/j.brs.2008.06.004 (2008).

42. Andrade, S. M., et al. Transcranial direct current stimulation over the primary motor vs prefrontal cortex in refractory chronic migraine: A pilot randomized controlled trial. Journal 
of the Neurological Sciences 378, 225-232. https://doi.org/10.1016/j.jns.2017.05.007 (2017).

43. O’Connell, N. E., Marston, L., Spencer, S., DeSouza, L. H., \& Wand, B. M. Cochrane Database of Systematic Reviews Non-invasive brain stimulation techniques for chronic pain (Review) Non-invasive brain stimulation techniques for chronic pain (Review). Cochrane Database of Systematic Reviews 4, CD008208.

https://doi.org/10.1002/14651858.CD008208.pub5 (2018).

44. Ali, M., et al. Differential efficacy of electric motor cortex stimulation and lesioning of the dorsal root entry zone for continuous vs paroxysmal pain after brachial plexus avulsion. Neurosurgery 68, 1252-1257. https://doi.org/10.1227/NEU.0b013e31820c04a9 (2011).

45. Rinaldi, P. C., Young, R. F., Albe-Fessard, D., \& Chodakiewitz, J. Spontaneous neuronal hyperactivity in the medial and intralaminar thalamic nuclei of patients with deafferentation pain. Journal of Neurosurgery 74, 415-421. https://doi.org/10.3171/jns.1991.74.3.0415 (1991).

46. Lefaucheur, J. P. Pain. Handbook of Clinical Neurology 116, 423-440. https://doi.org/10.1016/B978-0-444-53497-2.00035-8 (2013).

47. Fregni, F., et al. A randomized, sham-controlled, proof of principle study of transcranial direct current stimulation for the treatment of pain in fibromyalgia. Arthritis and Rheumatism 54, 3988-3998. https://doi.org/10.1002/art.22195 (2006).

48. Ngernyam, N., et al. The effects of transcranial direct current stimulation in patients with neuropathic pain from spinal cord injury. Clinical Neurophysiology 126, 382-390. https://doi.org/10.1016/j.clinph.2014.05.034 (2015).

49. Kim, Y. J., et al. Randomized, sham controlled trial of transcranial direct current stimulation for painful diabetic polyneuropathy. Annals of Rehabilitation Medicine 37 766776. https://doi.org/10.5535/arm.2013.37.6.766 (2013).

50. Yoon, E. J., et al. Transcranial Direct Current Stimulation to Lessen Neuropathic Pain After Spinal Cord Injury. Neurorehabilitation and Neural Repair 28, 250-259. https://doi.org/10.1177/1545968313507632 (2014).

51. Denny-Brown, D., Kirk, E. J., \& Yanagisawa, N. The tract of Lissauer in relation to sensory transmission in the dorsal horn of spinal cord in the macaque monkey. Journal of Comparative Neurology 151, 175-199. https://doi.org/https://doi.org/10.1002/cne.901510206 (1973).

52. Ovelmen-Levitt, J., Johnson, B., Bedenbaugh, P., \& Nashold Jr, B. S. Dorsal Root Rhizotomy and Avulsion in the Cat: A Comparison of Long Term Effects on Dorsal Horn Neuronal Activity. Neurosurgery 15, 921-927.

https://doi.org/https://doi.org/10.1097/00006123-198412000-00030 (1984).

53. Rodrigues, P. A., et al. Transcranial magnetic stimulation for the treatment of anxiety disorder. Neuropsychiatric Disease and Treatment 15, 2743-2761.

https://doi.org/10.2147/ndt.s201407 (2019). 


\section{Funding}

This study was supported by the Aging and Neuroscience Laboratory at Federal University of Paraíba, João Pessoa, Brazil.

\section{Author contributions}

EB: protocol/project development, data management, manuscript writing. WM: data collection, data management. CD, CM, RG, ER, UM: data collection. EM: manuscript editing and revision. SA: protocol/project development, data analysis, manuscript editing.

\section{Competing interests}

The authors declare no competing interests.

\section{Additional information}

Correspondence and requests for materials should be addressed to E.B.

Reprints and permissions information is available at www.nature.com/reprints. 


\section{Table 1. Demographic and clinical data at baseline (intent-to-treat population).}

\begin{tabular}{|c|c|c|}
\hline & Group $1(n=12)$ (active) & Group $2(n=8)($ sham $)$ \\
\hline Age $($ mean $\pm S D)$ & $33.53 \pm 9.99$ & $30.62 \pm 6.80$ \\
\hline $\begin{array}{r}\text { Gender, } \%(n) \\
\text { Male }\end{array}$ & $100(12)$ & $100(8)$ \\
\hline Injury time in months (mean $\pm S D)$ & $34.01 \pm 31.04$ & $44.44 \pm 36.86$ \\
\hline $\begin{array}{r}\text { Affected upper limb, \% (n) } \\
\text { Right } \\
\text { Left }\end{array}$ & $\begin{array}{l}58.33(7) \\
41.66(5)\end{array}$ & $\begin{array}{l}37.50(3) \\
62.50(5)\end{array}$ \\
\hline $\begin{array}{r}\text { Pain site, } \%(\boldsymbol{n}) \\
\text { Hand } \\
\text { Hand and forearm } \\
\text { Arm } \\
\text { Shoulder } \\
\text { Whole upper limb }\end{array}$ & $\begin{array}{r}58.33(7) \\
16.67(2) \\
8.33(1) \\
8.33(1) \\
8.33(1)\end{array}$ & $\begin{array}{l}37.50(3) \\
37.50(3) \\
12.50(1) \\
12.50(1)\end{array}$ \\
\hline $\begin{array}{r}\text { Number of affected dermatomes }(\boldsymbol{C 5}-\mathrm{TI})(\text { mean } \pm \text { SD) } \\
\text { Total affected } \\
\text { Hypoesthesia } \\
\text { Anesthesia }\end{array}$ & $\begin{array}{l}4.53 \pm 0.87 \\
2.35 \pm 1.54 \\
2.18 \pm 1.70\end{array}$ & $\begin{array}{l}4.63 \pm 0.74 \\
2.25 \pm 1.58 \\
2.25 \pm 1.39\end{array}$ \\
\hline $\begin{array}{r}\text { Affected myotomes (C5-T1), \% (n) } \\
\text { C5 } \\
\text { C6 } \\
C 7 \\
C 8 \\
T 1\end{array}$ & $\begin{array}{l}83.33(10) \\
100(12) \\
100(12) \\
91.67(11) \\
75.00(9)\end{array}$ & $\begin{array}{l}87.50(7) \\
100(8) \\
100(8) \\
100(8) \\
100(8)\end{array}$ \\
\hline Horner's syndrome, \% (n) & $75.00(9)$ & $75.00(6)$ \\
\hline $\begin{array}{r}\text { Presence of motor function, } \%(\boldsymbol{n}) \\
\text { Useful (MRC grade 3-4) } \\
\text { Residual (MRC grade 1-2) } \\
\text { Absent (MRC grade 0) }\end{array}$ & $\begin{array}{r}41.66(5) \\
50.00(6) \\
8.33(1)\end{array}$ & $\begin{array}{l}37.50(3) \\
50.00(4) \\
12.50(1)\end{array}$ \\
\hline $\begin{array}{r}\text { Location with motor function, \% (n) } \\
\text { Shoulder } \\
\text { Elbow } \\
\text { Wrist } \\
\text { Hand } \\
\text { Whole upper limb }\end{array}$ & $\begin{array}{r}50.00(6) \\
8.33(1) \\
16.67(2) \\
25.00(3) \\
8.33(1)\end{array}$ & $\begin{array}{l}50.00(4) \\
25.00(2) \\
25.00(2) \\
25.00(2)\end{array}$ \\
\hline $\begin{array}{r}\text { Medications used, \% (n) } \\
\text { Opioids } \\
\text { Antiepileptics } \\
\text { Antidepressants } \\
\end{array}$ & $\begin{array}{l}100(12) \\
83.33(10) \\
75.00(9)\end{array}$ & $\begin{array}{l}100(8) \\
75.00(6) \\
75.00(6)\end{array}$ \\
\hline $\begin{array}{r}\text { Physiotherapy, \% (n) } \\
\text { Yes, at the moment } \\
\text { No, but already did } \\
\text { Never did }\end{array}$ & $\begin{array}{l}66.67(8) \\
16.67(2) \\
16.67(2)\end{array}$ & $\begin{array}{l}87.5(7) \\
12.5(1)\end{array}$ \\
\hline$B D I($ mean $\pm S D)$ & $17.29 \pm 8.62$ & $20.88 \pm 7.18$ \\
\hline DN4 $($ mean $\pm S D)$ & $7.06 \pm 0.97$ & $7.00 \pm 1.20$ \\
\hline $\begin{array}{r}\text { VAS }(\text { mean } \pm \text { SD) } \\
\text { Continuous } \\
\text { Paroxysmal }\end{array}$ & $\begin{array}{l}5.59 \pm 2.27 \\
8.29 \pm 2.08\end{array}$ & $\begin{array}{l}5.13 \pm 1.89 \\
8.00 \pm 2.07\end{array}$ \\
\hline $\begin{array}{r}\text { MPQ (mean } \pm \text { SD }) \\
\text { No. of descriptors } \\
\text { Pain index }\end{array}$ & $\begin{array}{l}19.53 \pm 1.28 \\
42.82 \pm 11.92\end{array}$ & $\begin{array}{l}19.75 \pm 0.71 \\
41.38 \pm 10.68\end{array}$ \\
\hline $\begin{array}{r}\text { STAI }(\text { mean } \pm \text { SD }) \\
\text { Trait } \\
\text { State }\end{array}$ & $\begin{array}{l}48.88 \pm 8.28 \\
44.71 \pm 5.54\end{array}$ & $\begin{array}{l}50.88 \pm 7.30 \\
42.63 \pm 4.03\end{array}$ \\
\hline$S F-36($ mean $\pm S D)$ & $335.30 \pm 154.87$ & $320.25 \pm 181.60$ \\
\hline
\end{tabular}

Clinical and sociodemographic characteristics at baseline did not differ between the groups.

BDI Beck Depression Inventory, DN4 DN4 questionnaire, VAS Visual Analogic Scale, MPQ McGill Pain Questionnaire, STAI State-Trait Anxiety Inventory, SF-36 SF-36 Quality of Life Questionnaire, MRC Medical Research Council. 
Table 2. Side effects observed after stimulation sessions.

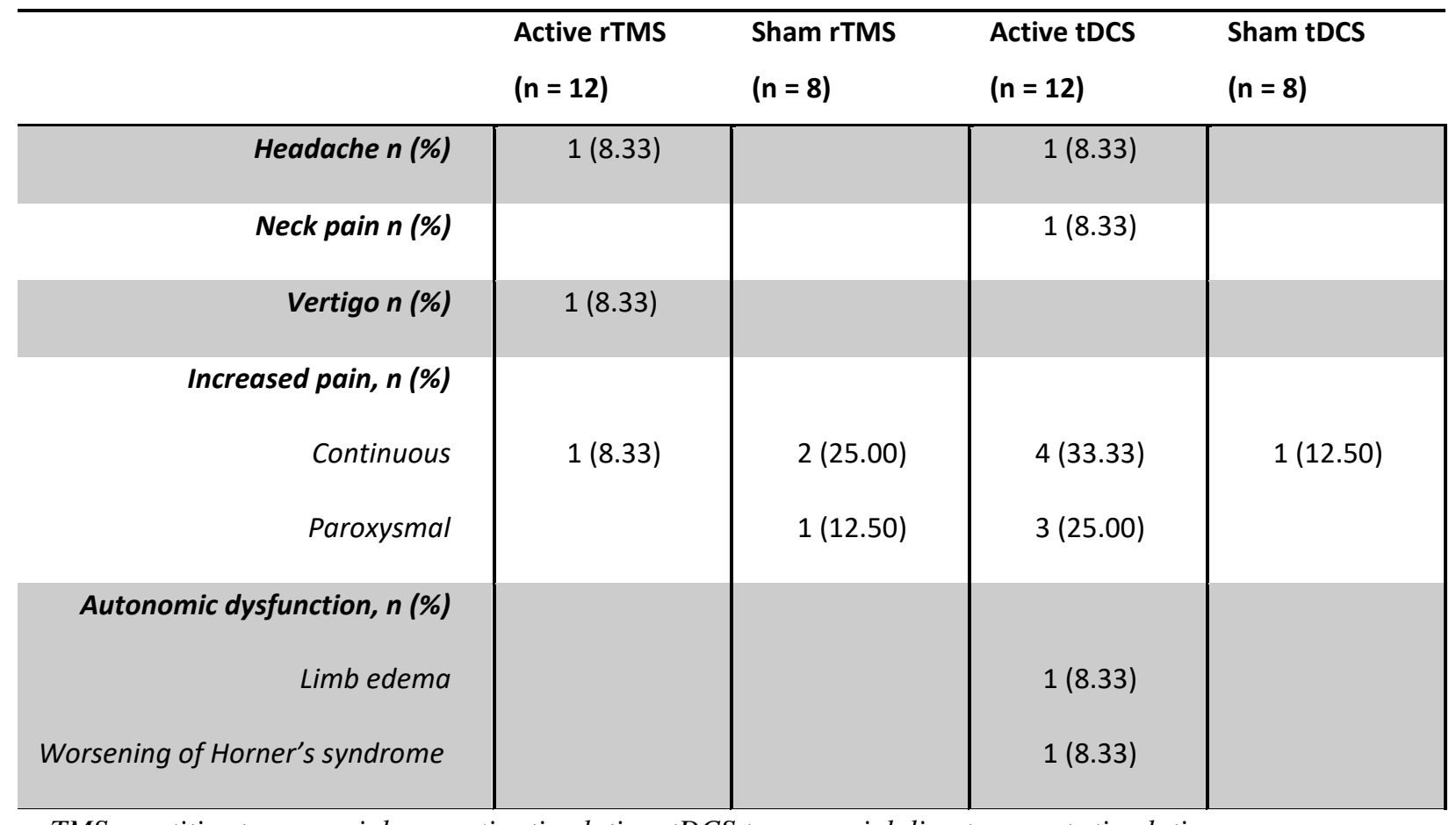

rTMS repetitive transcranial magnetic stimulation, tDCS transcranial direct-current stimulation. 


\section{Figure captions}

Figure 1. Study Design. The treatment protocol included 2 stimulation blocks separated by a 30-day interval. Each block consisted of 5 sessions for 5 consecutive days, during which each patient received repetitive transcranial magnetic stimulation (rTMS) or transcranial directcurrent stimulation (tDCS) for 30 minutes. Each patient underwent 10 stimulation sessions in all (2 series of 5 active rTMS or tDCS sessions or 2 series of 5 sham rTMS or tDCS sessions). Assessments took place immediately before the first stimulation (T0), after the 5th consecutive stimulation session (T1) and 30 days after (T2). $M 1$ primary motor cortex.

Figure 2. Patient disposition and CONSORT flowchart. ITT intent-to-treat.

Figure 3. Effects of active repetitive transcranial magnetic stimulation (rTMS), active transcranial direct-current stimulation (tDCS), and sham stimulation on average continuous pain intensity (VAS). The scores were obtained before the first stimulation session (T0), after the 5th consecutive stimulation session (T1) and 30 days after (T2). For the sake of simplicity, and as the effects of sham rTMS and sham tDCS were remarkably similar regardless of the order in which they were conducted, we present the mean values grouped into a single sham-stimulation group. Error bars indicate standard error of the mean. VAS Visual Analogic Scale.

Figure 4. Effects of active repetitive transcranial magnetic stimulation (rTMS), active transcranial direct-current stimulation (tDCS), and sham stimulation on average paroxysmal pain intensity (VAS). The scores were obtained before the first stimulation session (T0), after the 5th consecutive stimulation session (T1) and 30 days after (T2). For the sake of simplicity, and as the effects of sham rTMS and sham tDCS were remarkably similar regardless of the order in which they were conducted, we present the mean values grouped into a single sham-stimulation group. Error bars indicate standard error of the mean. VAS Visual Analogic Scale.

Figure 5. Effects of active repetitive transcranial magnetic stimulation (rTMS), active transcranial direct-current stimulation (tDCS), and sham stimulation on average multidimensional aspect of pain (MPQ). The scores were obtained before the first stimulation session (T0), after the 5th consecutive stimulation session (T1) and 30 days after (T2). For the sake of simplicity, and as the effects of sham rTMS and sham tDCS were remarkably similar regardless of the order in which they were conducted, we present the mean values grouped into a single sham-stimulation group. Error bars indicate standard error of the mean. $M P Q$ McGill Pain Questionnaire.

Figure 6. Effects of active repetitive transcranial magnetic stimulation (rTMS), active transcranial direct-current stimulation (tDCS), and sham stimulation on average anxiety state (STAI-S). The scores were obtained before the first stimulation session (T0), after the 5th consecutive stimulation session (T1) and 30 days after (T2). For the sake of simplicity, and as the effects of sham rTMS and sham tDCS were remarkably similar regardless of the order in which they were conducted, we present the mean values grouped into a single sham-stimulation group. Error bars indicate standard error of the mean. STAI-S State-Trait Anxiety Inventory (state subscale). 
Figure 1 (Study Design)

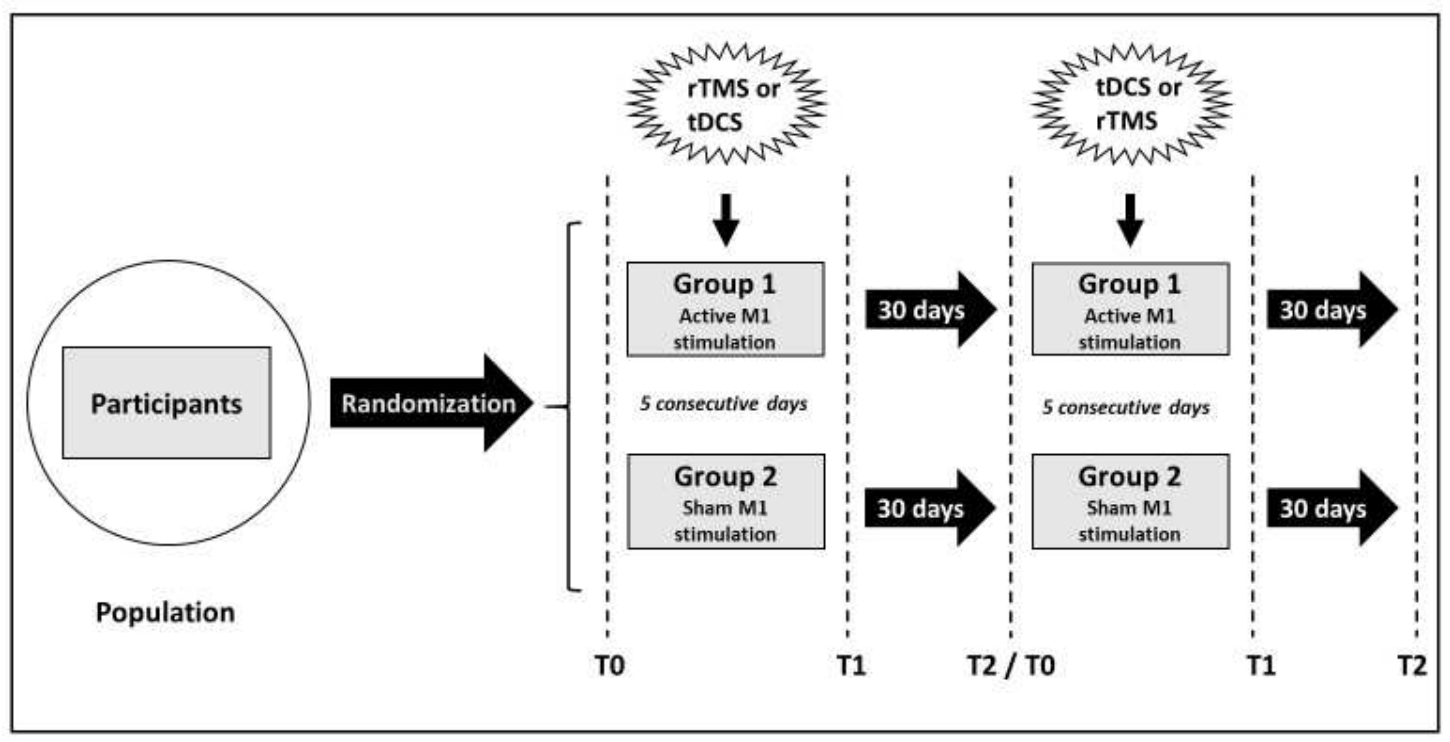


Figure 2 (CONSORT Flowchart)

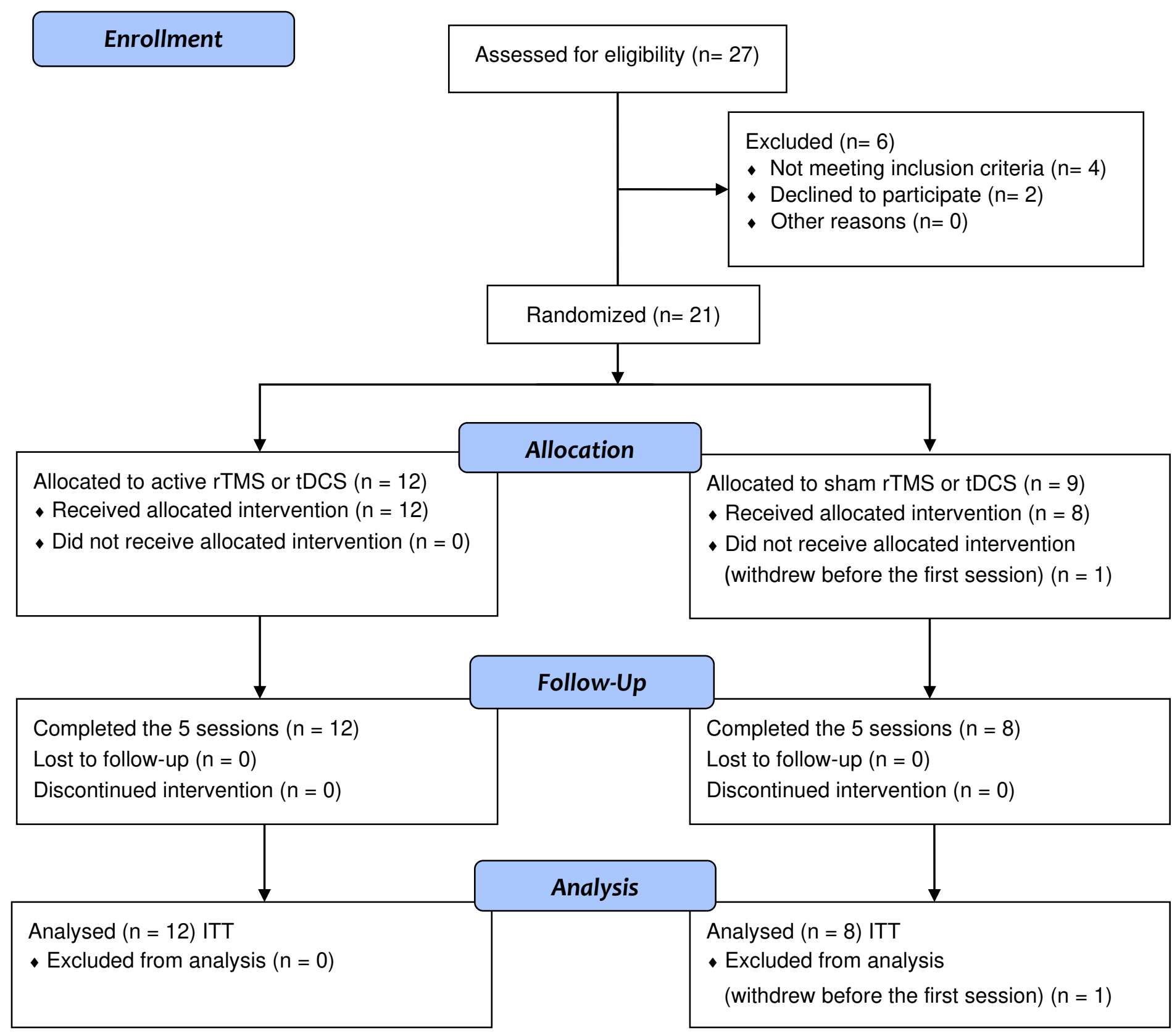


Figure 3 (VAS Results - Continuous Pain)

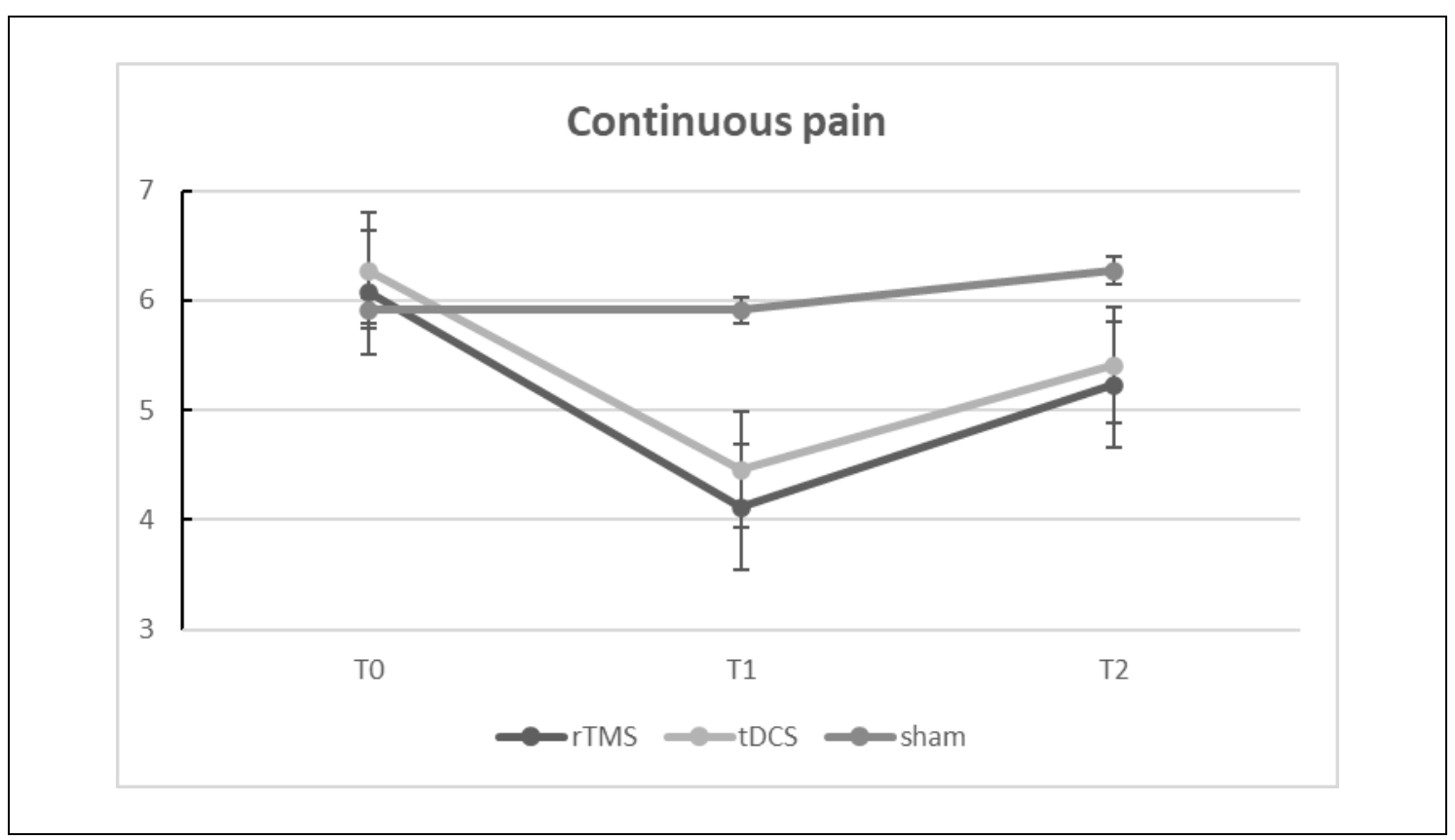


Figure 4 (VAS Results - Paroxysmal Pain)

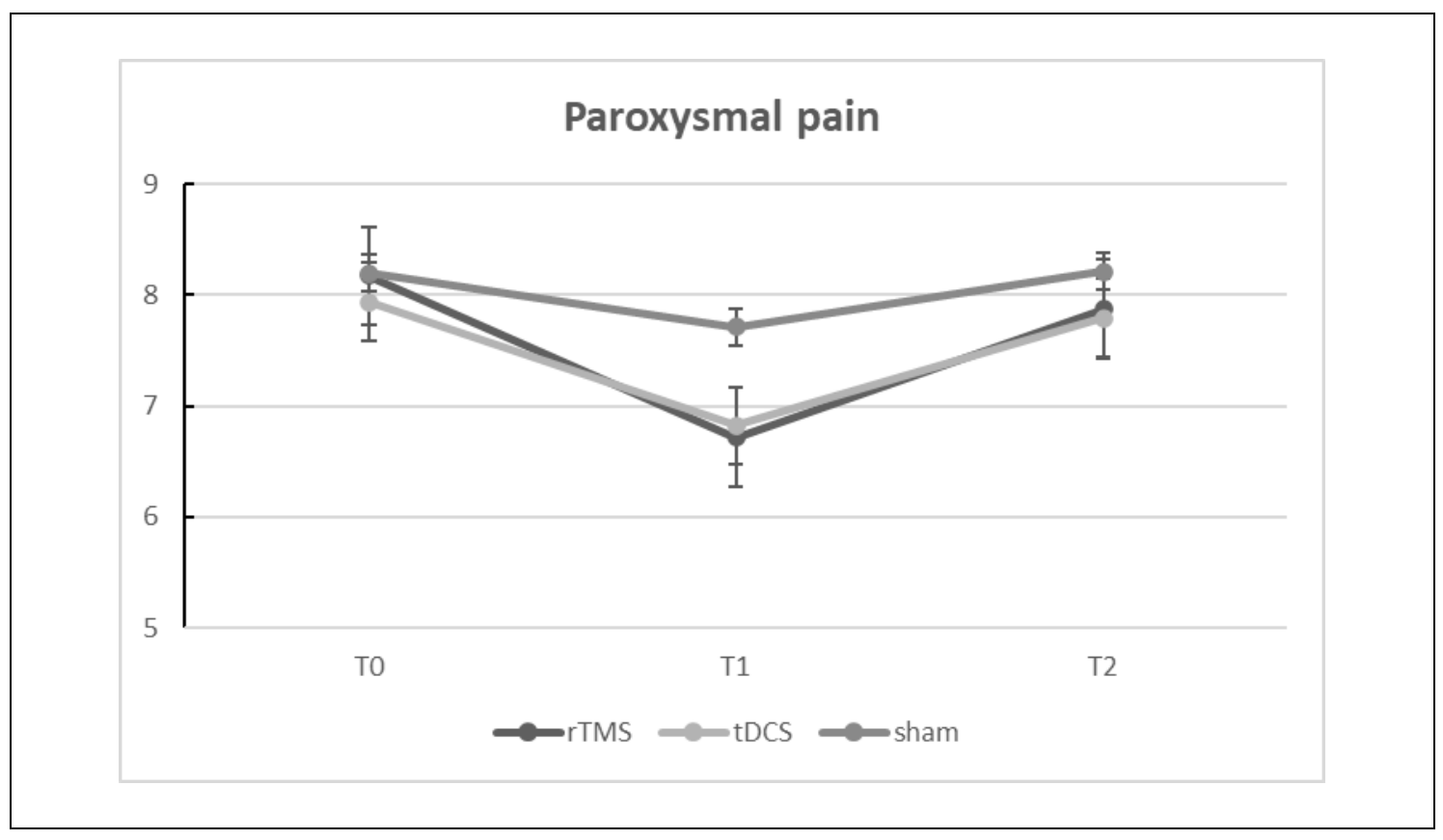


Figure 5 (MPQ Results)

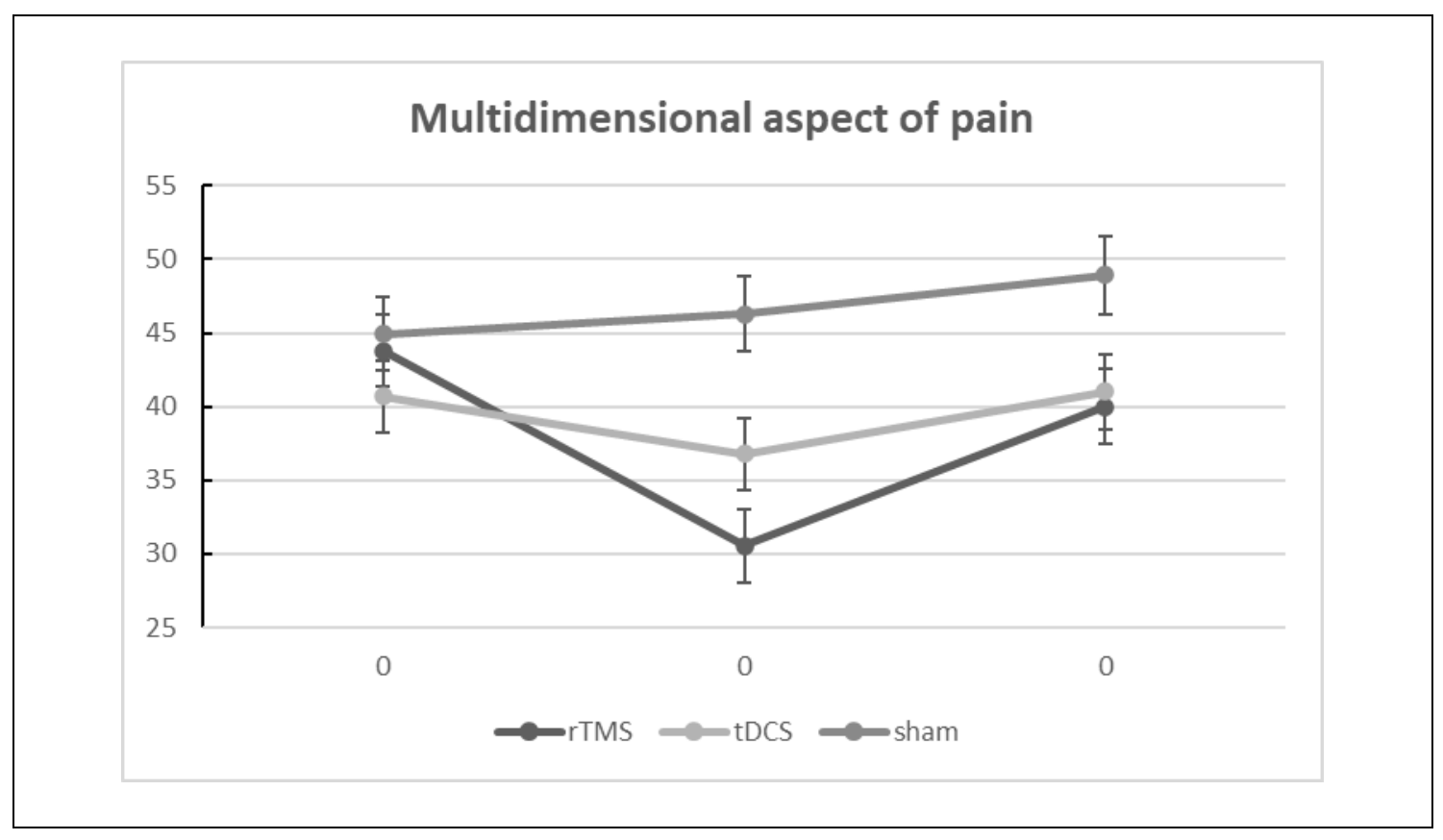


Figure 6 (STAI-S Results)

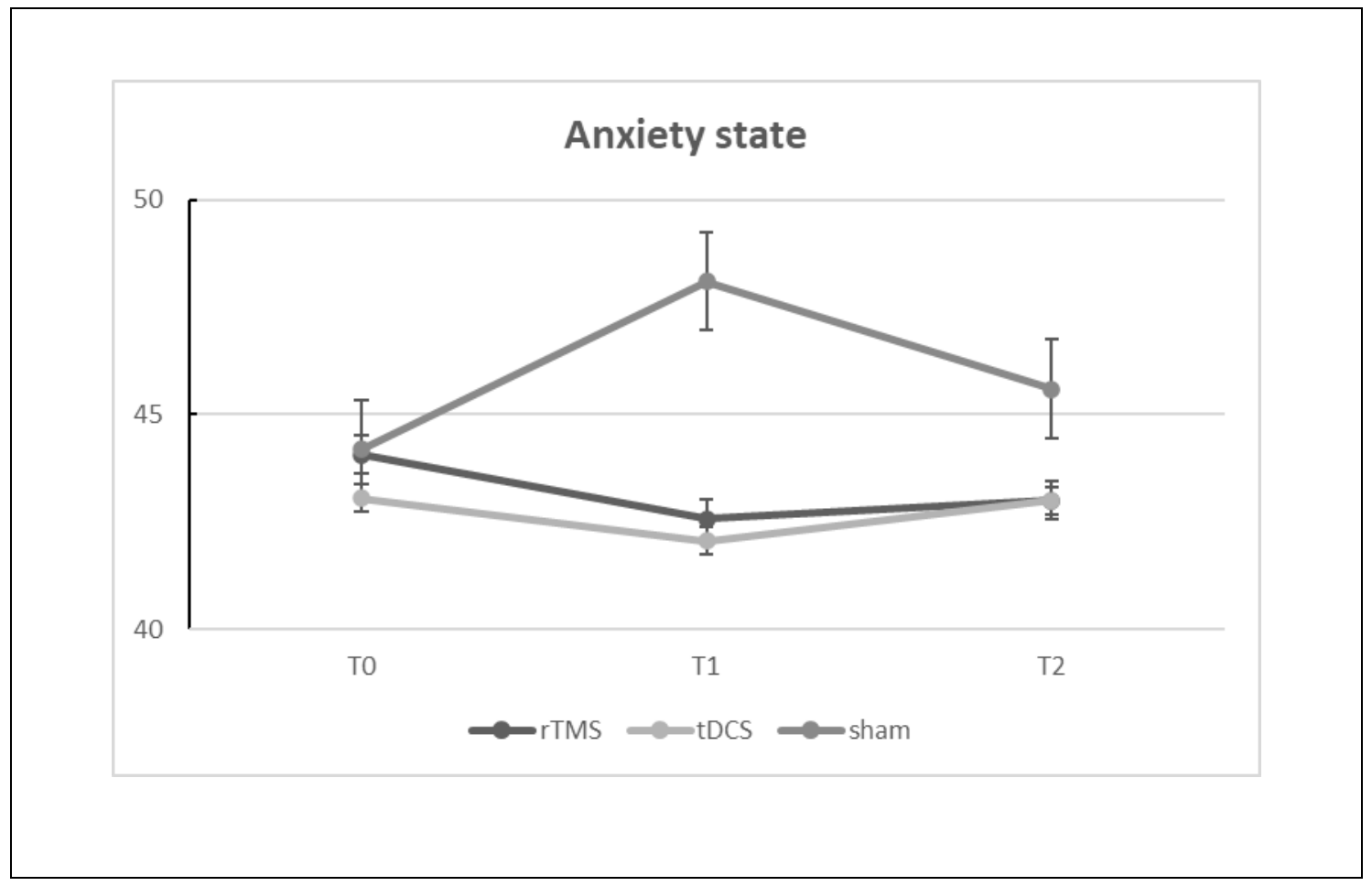




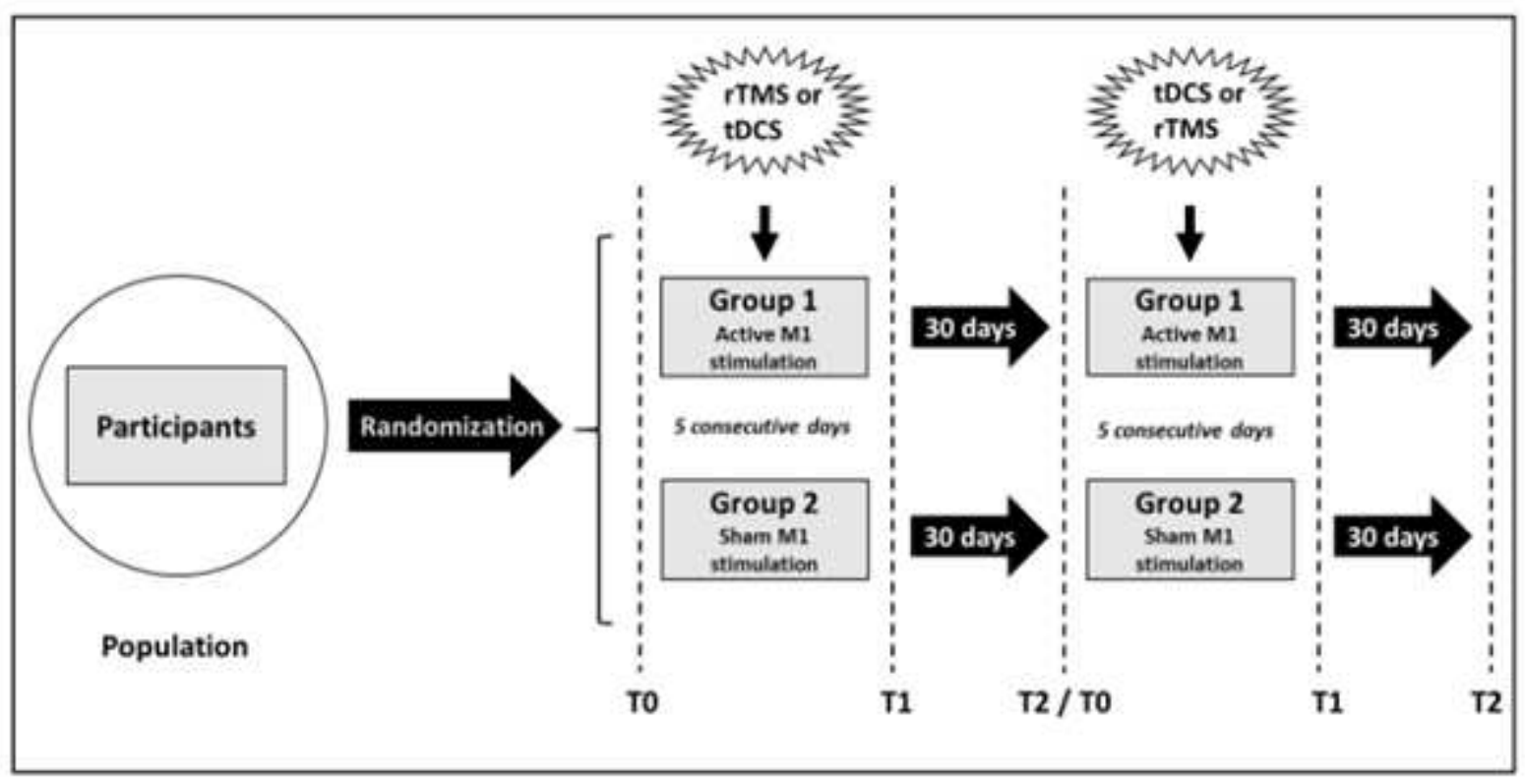

Figure 1

Study Design. The treatment protocol included 2 stimulation blocks separated by a 30-day interval. Each block consisted of 5 sessions for 5 consecutive days, during which each patient received repetitive transcranial magnetic stimulation (rTMS) or transcranial direct-current stimulation (tDCS) for 30 minutes. Each patient underwent 10 stimulation sessions in all (2 series of 5 active rTMS or tDCS sessions or 2 series of 5 sham rTMS or tDCS sessions). Assessments took place immediately before the first stimulation (T0), after the 5th consecutive stimulation session (T1) and 30 days after (T2). M1 primary motor cortex. 


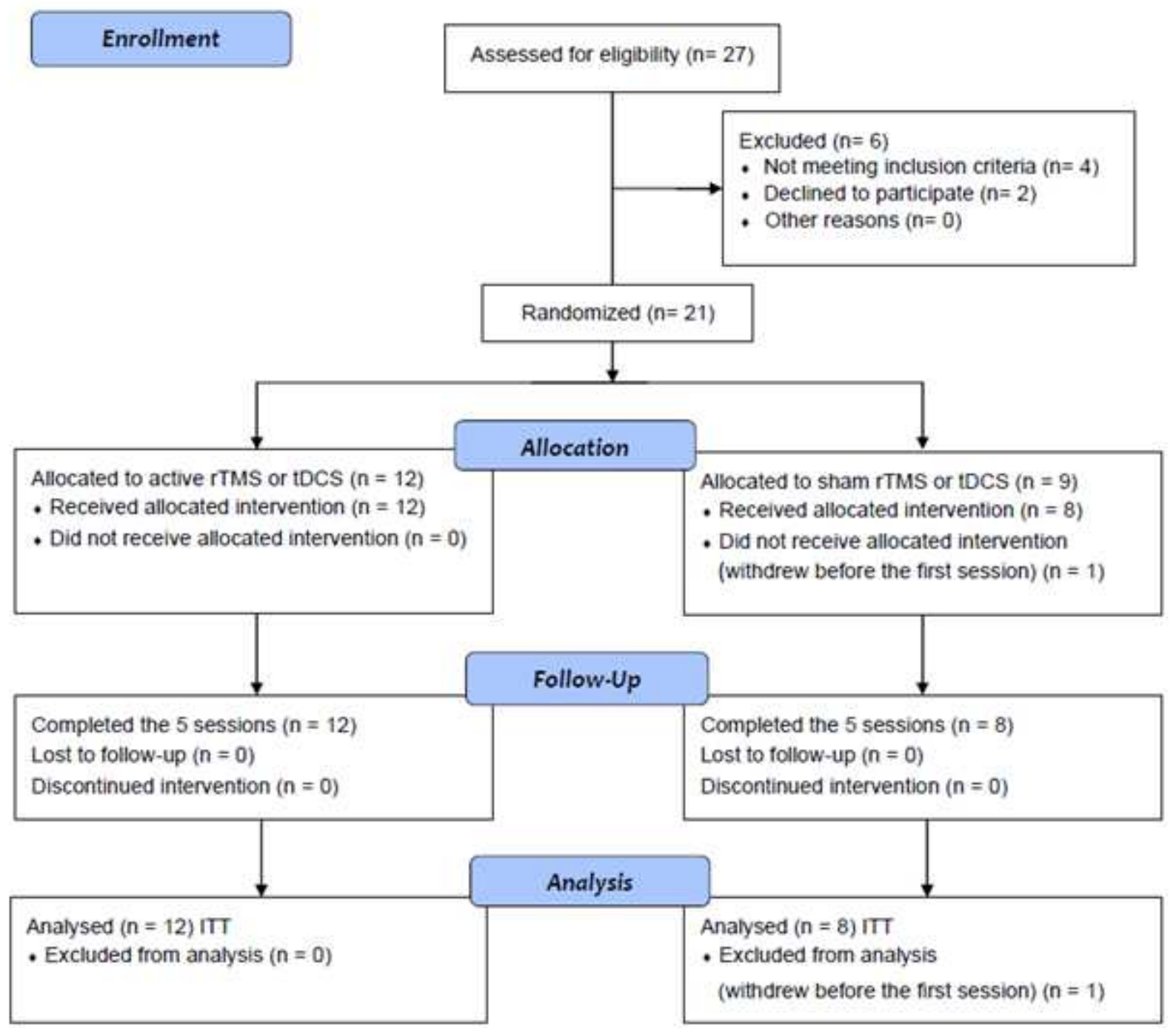

Figure 2

Patient disposition and CONSORT flowchart. ITT intent-to-treat. 


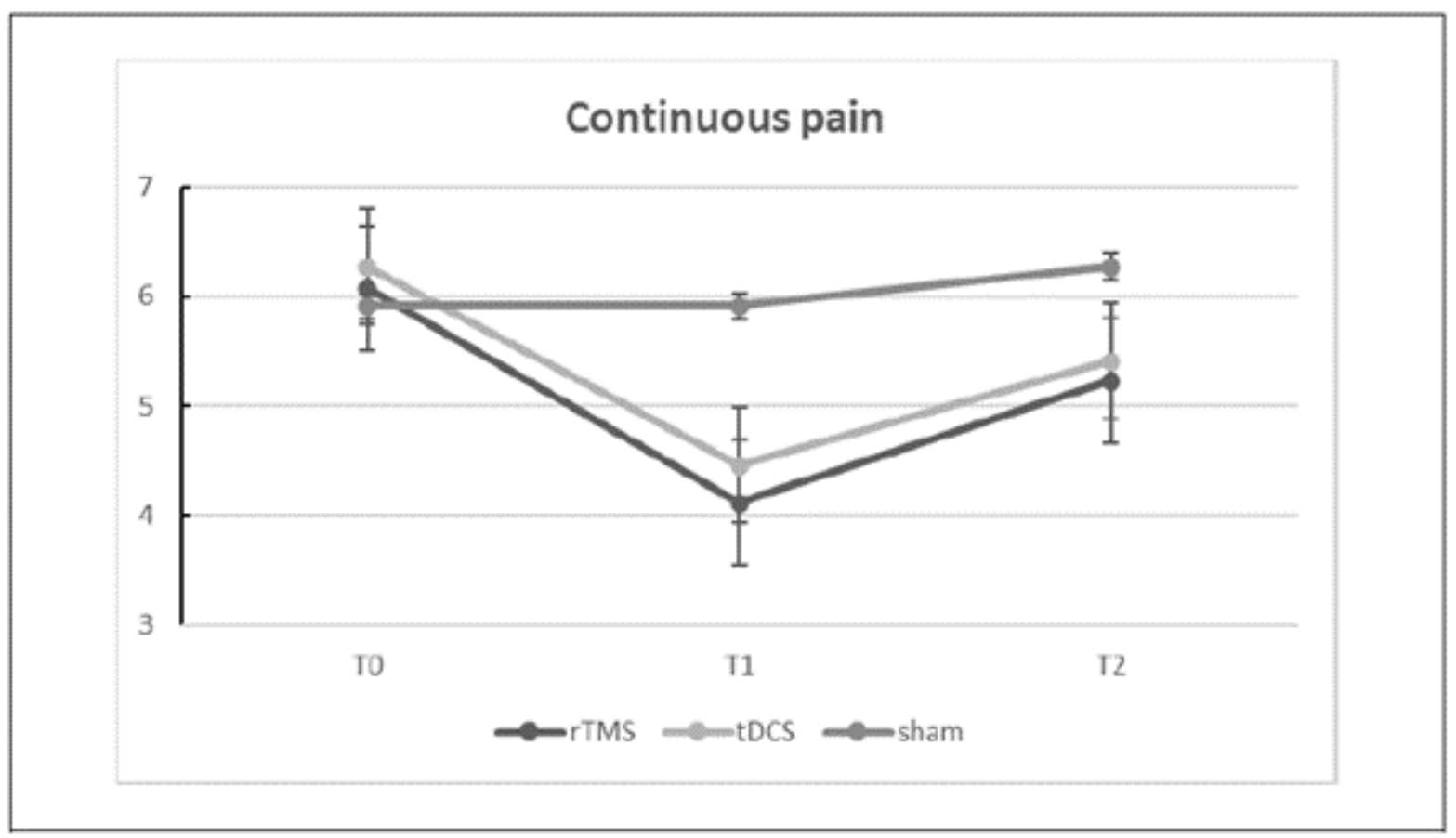

\section{Figure 3}

Effects of active repetitive transcranial magnetic stimulation (rTMS), active transcranial direct-current stimulation (tDCS), and sham stimulation on average continuous pain intensity (VAS). The scores were obtained before the first stimulation session (T0), after the 5th consecutive stimulation session (T1) and 30 days after (T2). For the sake of simplicity, and as the effects of sham rTMS and sham tDCS were remarkably similar regardless of the order in which they were conducted, we present the mean values grouped into a single sham-stimulation group. Error bars indicate standard error of the mean. VAS Visual Analogic Scale.

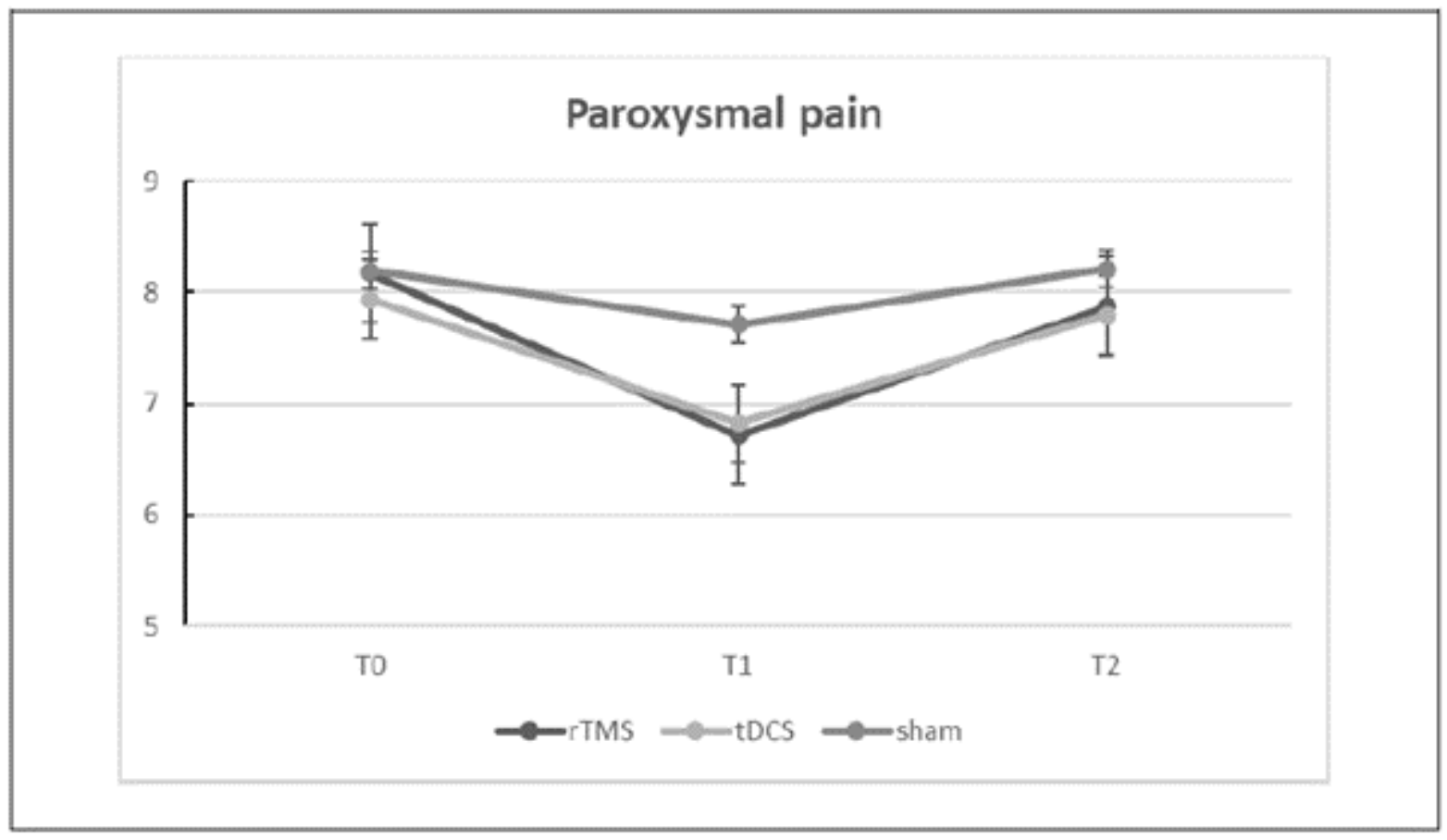




\section{Figure 4}

Effects of active repetitive transcranial magnetic stimulation (rTMS), active transcranial direct-current stimulation (tDCS), and sham stimulation on average paroxysmal pain intensity (VAS). The scores were obtained before the first stimulation session (T0), after the 5th consecutive stimulation session (T1) and 30 days after (T2). For the sake of simplicity, and as the effects of sham rTMS and sham tDCS were remarkably similar regardless of the order in which they were conducted, we present the mean values grouped into a single sham-stimulation group. Error bars indicate standard error of the mean. VAS Visual Analogic Scale.

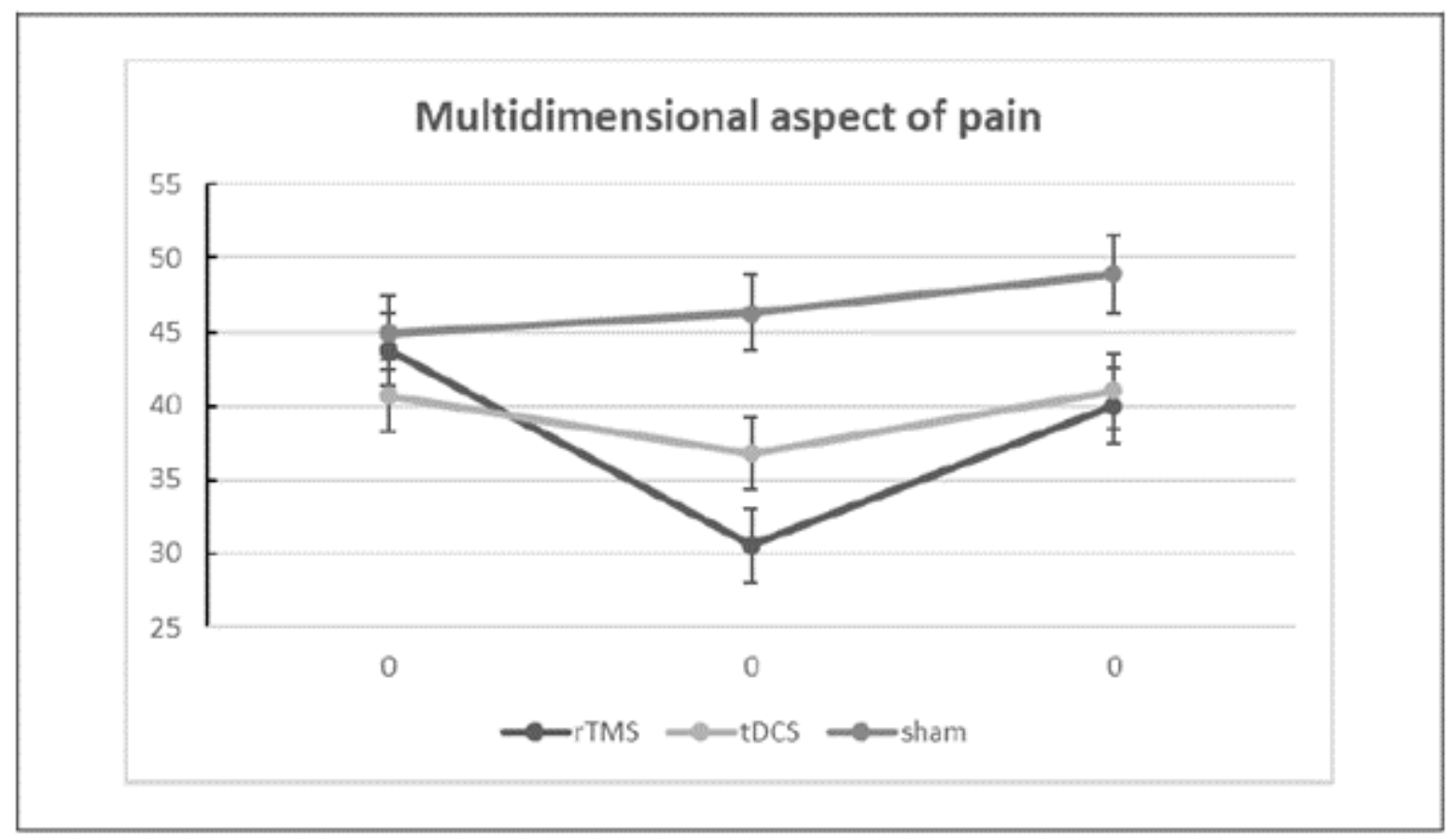

\section{Figure 5}

Effects of active repetitive transcranial magnetic stimulation (rTMS), active transcranial direct-current stimulation (tDCS), and sham stimulation on average multidimensional aspect of pain (MPQ). The scores were obtained before the first stimulation session (T0), after the 5th consecutive stimulation session (T1) and 30 days after (T2). For the sake of simplicity, and as the effects of sham rTMS and sham tDCS were remarkably similar regardless of the order in which they were conducted, we present the mean values grouped into a single sham-stimulation group. Error bars indicate standard error of the mean. MPQ McGill Pain Questionnaire. 


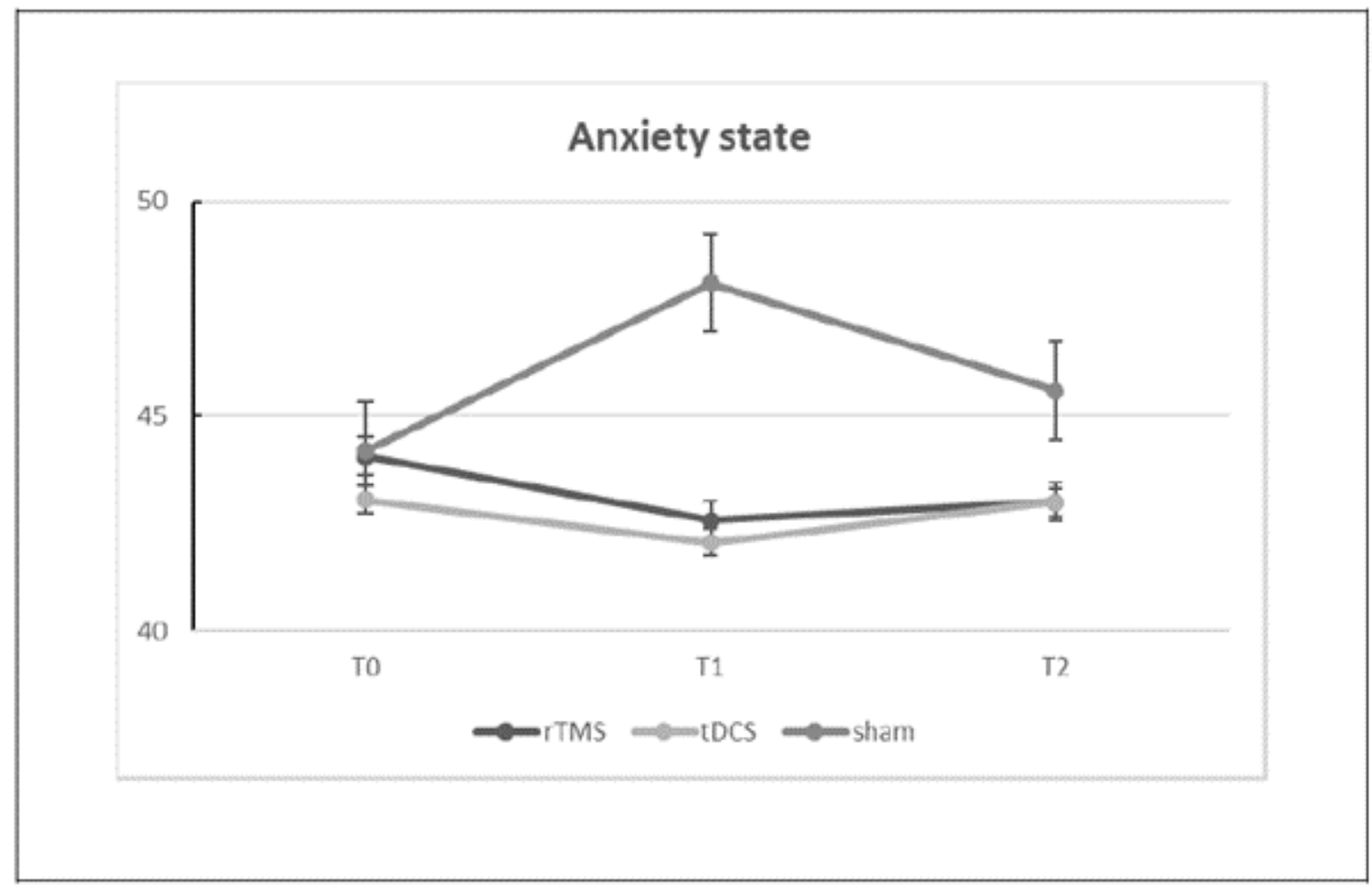

Figure 6

Effects of active repetitive transcranial magnetic stimulation (rTMS), active transcranial direct-current stimulation (tDCS), and sham stimulation on average anxiety state (STAI-S). The scores were obtained before the first stimulation session (T0), after the 5th consecutive stimulation session (T1) and 30 days after (T2). For the sake of simplicity, and as the effects of sham rTMS and sham tDCS were remarkably similar regardless of the order in which they were conducted, we present the mean values grouped into a single sham-stimulation group. Error bars indicate standard error of the mean. STAI-S State-Trait Anxiety Inventory (state subscale). 